\title{
Synthesis and elastic behaviour of borate glass doped with high tellurite content
}

\begin{abstract}
A systematic series of quality binary glass system of $(\mathrm{x}) \mathrm{TeO} 2)-(1-\mathrm{x}) \mathrm{B} 2 \mathrm{O} 3$ with $\mathrm{x}=60$ to 80 (wt. \%) were successfully synthesized by the rapid quenching method. The densities of each glass samples were determined using Archimedes method with acetone as a floatation medium. The molar volume have been estimated and analyzed for borate glasses doped with tellurite. Ultrasonics methods have been used to study the elastic properties of TeO2B2O3 glasses where the sound wave velocities have been measured in each glass samples at a frequency of $15 \mathrm{MHz}$ and at room temperature. The velocities, both longitudinal and transverse, increase linearly with increasing of $\mathrm{TeO} 2$ content in a borate glass network. Their elastic moduli such as longitudinal, Young's, bulk and shear modulus have been calculated as a function of $\mathrm{TeO} 2$ concentration. Poisson's ratio and Debye temperature were also found to increase nonlinearly with $\mathrm{TeO} 2$ concentration. The glass transition temperature were determined by the differential thermogravimetric analysis at heating rate of $20 \mathrm{~K}$ min- 1 . However the glass transition temperature ( $\mathrm{Tg}$ ) slowly decrease as more tellurite is added into the borate glass network. (C) 2006 Asian Network for Scientific Information.
\end{abstract}

Keyword: Borate; Elastic properties; Glass; Telurrite; Thermal behaviour 